\title{
Tetraquark bound states in a constituent quark model and the nature of the $a_{0}(980)$ and $f_{0}(980)$
}

\author{
J. Vijande ${ }^{(1)}$, F. Fernández ${ }^{(1)}$, A. VAlCARCe ${ }^{(1)}$ And B. \\ Silvestre-Brac (2)
}

(1) Grupo de Física Nuclear

Universidad de Salamanca, E-37008 Salamanca, Spain

(2) Institut des Sciences Nucléaires

53 Av. des Martyrs, F-38026 Grenoble cedex, France

PACS numbers: 12.39.-x,13.75.Lb

The pseudoscalar and vector meson ground states and their low-energy excited states have been understood as $q \bar{q}$ pairs. However, the structure of the scalar mesons, $\left(J^{P C}=0^{++}\right)$, remains controversial nowadays. In the naive quark model, to construct a positive parity state requires a unit of angular momentum in a $q \bar{q}$ pair. Apparently, this takes an energy around 1 $\mathrm{GeV}$ since similar meson states $\left(1^{++}\right.$and $\left.2^{++}\right)$lie above $1.2 \mathrm{GeV}$. However a more complicated structure, like $q^{2} \bar{q}^{2}$, suggested twenty years ago by Jaffe [1] can couple to $0^{++}$without orbital excitation and therefore could be a serious candidate to explain the structure of the lightest scalar mesons.

In this work we study tetraquark bound states in the framework of the constituent quark model of Ref. [2], which has been used for the description of non-strange two- and three-baryon systems and later on applied to the hadron spectra. The model is based on the idea that between the scale of chiral symmetry breaking and the confinement scale, QCD may be formulated for the light quark sector as an effective theory of constituent quarks interacting through gluon and Goldstone boson exchanges. For the heavy sector chiral symmetry is explicitly broken through the current quark masses and, as a consequence, the interaction reduces to confinement and gluon terms. Expressions of the interaction can be found elsewhere [2].

We will focus in two particular configurations. The first one will be the light-heavy states, $[(q q)(\bar{Q} \bar{Q})]$, since they are the most prominent candidates to be bound under the strong interaction $[3,4]$. The second one will be those tetraquarks with the same quantum numbers as the scalar mesons [5]. We solve the Schrödinger equation using a variational method where the spatial trial wave function is a linear combination of gaussians 


$$
\Psi \propto e^{-a_{i} \vec{x}^{2}-b_{i} \vec{y}^{2}-c_{i} \vec{z}^{2}-d_{i} \vec{x} \cdot \vec{y}-e_{i} \vec{x} \cdot \vec{z}-f_{i} \vec{y} \cdot \vec{z}}
$$

$\vec{x}, \vec{y}$ and $\vec{z}$ being the tetraquark Jacobi coordinates and $a_{i}, b_{i}, c_{i}, d_{i}, e_{i}$ and $f_{i}$ the variational parameters.

The calculation is done using the full color-space wave function, including the two possible color singlets, $\{\overline{3} 3\}$ and $\{6 \overline{6}\}$, despite of the fact that the $\{6 \overline{6}\}$ component has been usually neglected [4]. The spatial exchange terms (those depending, for example, on $\vec{y} \cdot \vec{z}$ in Eq. (1)) have also been usually neglected [4]. If one makes such approximation then, $[(q q)(\bar{Q} \bar{Q})]$ $(I, S)=(0,0)$ and $(I, S)=(0,2)$ states would be forbidden [6].

We checked the importance of the spatial exchange terms by comparing the results obtained with and without them. As a general trend we found a contribution to the binding energy lower than $0.5 \%$. This is why the spatial exchange terms will be only used when studying the light-heavy states with $(I, S)=(0,0)$ or $(I, S)=(0,2)$, and will be neglected in the other cases. Most part of the model parameters have been fixed when studying the $N N$ interaction and the baryon spectrum. The remaining ones are fixed by adjusting the meson spectrum [7].

In Table 1 we present our results for the different flavor contents of the light-heavy systems. We show the total energy $\left(E_{T}\right)$ and the energy difference with respect to the lowest possible threshold, $\Delta E=E_{T}(q q \bar{Q} \bar{Q})-$ $E_{1}(q \bar{Q})-E_{2}(q \bar{Q})$. Our calculation predicts only five stable tetraquarks. In particular one can see how the higher binding energies are found in the $(I, S)=(0,1)$ channel, as has been usually predicted [4]

The relative importance of both color channels is illustrated in Table 2. Being small in all cases, one can see how the contribution of the $\{6 \overline{6}\}$ color channel decreases when increasing the heavy quark mass. We also observe a dependence on the spin of the system. This behavior can be easily understood taken into account that the one-gluon exchange is the responsible for the color channel mixing and it depends on the inverse of the quark masses and on the total spin of the system.

Concerning the possible tetraquark structure of the scalar mesons we have studied systems with the same quantum numbers of the $a_{0}(980)$ and the $f_{0}(980)$. These mesons are $J^{P C}=0^{++}$with strangeness 0 , so they would correspond to a tetraquark with $(I, S)=(0,0)$ for the $f_{0}$, and $(I, S)=(1,0)$ for the $a_{0}$. Experimental observations $[5,8]$ support the idea that the $f_{0}$ has a $s \bar{s}$ component, and therefore we will consider as first approach a $[(q s)(\bar{q} \bar{s})]$ structure for the $f_{0}$ and $a_{0}$.

The obtained energies are the following: $E(I=0)=1282 \mathrm{MeV}$ and $E(I=1)=1275 \mathrm{MeV}$. We can see see how the energies are almost the same, as experimentally measured, but the absolute value is too high for claiming that these states correspond to the experimental $a_{0}$ an $f_{0}$. 


\begin{tabular}{|cc|cccccc|}
\hline & & \multicolumn{6}{|c|}{$(\mathrm{I}, \mathrm{S})$} \\
& & $(0,0)$ & $(1,0)$ & $(0,1)$ & $(1,1)$ & $(0,2)$ & $(1,2)$ \\
\hline$[(q q)(\bar{s} \bar{s})]$ & $E_{T}$ & 2185 & 1660 & 1399 & 1810 & 2676 & 1894 \\
& $\Delta E$ & +1188 & +663 & +63 & +474 & +1002 & +220 \\
{$[(q q)(\bar{c} \bar{c})]$} & $E_{T}$ & 4175 & 3947 & 3556 & 3975 & 4745 & 4001 \\
& $\Delta E$ & +438 & +210 & -254 & +165 & +862 & +118 \\
{$[(q q)(\bar{b} \bar{b})]$} & $E_{T}$ & 10677 & 10493 & 10072 & 10498 & 11251 & 10509 \\
& $\Delta E$ & +120 & -64 & -509 & -83 & +646 & -96 \\
\hline
\end{tabular}

Table 1. Total $\left(E_{T}\right)$ and binding energy $(\Delta E)$ in $\mathrm{MeV}$ for the light-heavy sector.

\begin{tabular}{|c|cc|cc|}
\hline & \multicolumn{2}{|c|}{$(\mathrm{I}, \mathrm{S})=(1,0)$} & \multicolumn{2}{c|}{$(\mathrm{I}, \mathrm{S})=(0,1)$} \\
& $\{\overline{3} 3\}$ & $\{\overline{3} 3\}$ and $\{6 \overline{6}\}$ & $\{\overline{3} 3\}$ & $\{\overline{3} 3\}$ and $\{6 \overline{6}\}$ \\
\hline$[(q q)(\bar{s} \bar{s})]$ & 1762 & 1660 & 1422 & 1399 \\
{$[(q q)(\bar{c} \bar{c})]$} & 3962 & 3947 & 3558 & 3556 \\
{$[(q q)(\bar{b} \bar{b})]$} & 10493 & 10493 & 10072 & 10072 \\
\hline
\end{tabular}

Table 2. Contribution of the different color channels in $\mathrm{MeV}$.

The origin of this discrepancy may be associated to our simplified ansatz for the confining interaction. We have assumed that confinement has only two-body potentials, however in the tetraquark systems three- and fourbody terms are expected to play a role. To take into account these contributions, not included in our model, we have modified the confinement parameter, $a_{c}^{\text {Tetraquark }}=0.765 * a_{c}^{\text {Meson }}$, obtaining $E(I=0)=987 \mathrm{MeV}$ and $E(I=1)=981 \mathrm{MeV}$, both nearly degenerated and with the right absolute value.

As a summary, the model studied predicts a reasonable description of tetraquark states in the light-heavy sector and supports the hypothesis of the $a_{0}$ and $f_{0}$ mesons having a tetraquark structure.

\section{REFERENCES}

[1] R.J. Jaffe. Phys. Rev. D15 (1977) 267.

[2] F. Fernández et al. J. Phys. G19 (1993) 2013.

[3] B. Silvestre-Brac, C. Semay. Z. Phys. C57 (1993) 273.

[4] S. Pepin et al. Phys. Lett. B393 (1997) 119.

[5] N.N. Achasov. Nucl. Phys. A675 (2000) 279.

[6] B. Silvestre-Brac. Phys. Rev. D46 (1992) 2179.

[7] J. Weinstein, N. Isgur. Phys. Rev. D27 (1983) 588.

[8] F. De Fazio, M.R. Pennington. Phys. Lett. B521 (2001) 15. 